\title{
QUASI-STATIONARY DISTRIBUTIONS FOR BIRTH-DEATH PROCESSES WITH KILLING
}

\author{
PAULINE COOLEN-SCHRIJNER AND ERIK A. VAN DOORN
}

Received 9 January 2006; Revised 9 June 2006; Accepted 28 July 2006

The Karlin-McGregor representation for the transition probabilities of a birth-death process with an absorbing bottom state involves a sequence of orthogonal polynomials and the corresponding measure. This representation can be generalized to a setting in which a transition to the absorbing state (killing) is possible from any state rather than just one state. The purpose of this paper is to investigate to what extent properties of birth-death processes, in particular with regard to the existence of quasi-stationary distributions, remain valid in the generalized setting. It turns out that the elegant structure of the theory of quasi-stationarity for birth-death processes remains largely intact as long as killing is possible from only finitely many states. In particular, the existence of a quasi-stationary distribution is ensured in this case if absorption is certain and the state probabilities tend to zero exponentially fast.

Copyright (c) 2006 P. Coolen-Schrijner and E. A. van Doorn. This is an open access article distributed under the Creative Commons Attribution License, which permits unrestricted use, distribution, and reproduction in any medium, provided the original work is properly cited.

\section{Introduction}

By a famous result of Karlin and McGregor [5], the transition probabilities of a birthdeath process on (a subset of) the integers can, under suitable conditions, be expressed in terms of a sequence of orthogonal polynomials and their orthogonalizing measure. This representation has led to detailed knowledge of many specific birth-death processes and to considerable insight into the behaviour of birth-death processes in general.

Evidently, it is of interest to investigate to what extent properties of birth-death processes retain their validity if one allows more general transition structures. Such investigations are usually hampered by the fact that the orthogonal-polynomial representation for the transition probabilities and the analytical tools that go with it are no longer available. The class of processes which is the subject of this article-and which comprises an outwardly mild generalization of birth-death processes-does not have this drawback. At

Hindawi Publishing Corporation

Journal of Applied Mathematics and Stochastic Analysis

Volume 2006, Article ID 84640, Pages 1-15

DOI 10.1155/JAMSA/2006/84640 
the same time, the class is interesting because it displays several of the phenomena that occur beyond the setting of the pure birth-death process.

Concretely, we will consider birth-death processes on the set $\{-1,0,1, \ldots\}$, with -1 being an absorbing bottom state, and the additional feature that absorption in one step (killing) may occur from any state rather than just one state. In particular, the existence and the shape of quasi-stationary distributions (initial distributions with the property that the state distribution of the process, conditional on nonabsorption, is constant over time) will be our main concern. It has recently been shown in [11] that an orthogonalpolynomial representation for the transition probabilities remains valid in this setting, so that the orthogonal-polynomial toolbox may be used to analyse the behaviour of such a process. In fact, the existence of quasi-stationary distributions will be shown to depend on the asymptotic behaviour of the orthogonal polynomials involved.

Quasi-stationarity for birth-death processes with killing has recently been studied in [4] in a discrete-time setting. In this setting, the analysis is simpler because the asymptotic behaviour of the pertinent orthogonal polynomials plays a less restrictive role. A recent paper by Steinsaltz and Evans [8] addresses related problems in the setting of diffusions with killing.

The remainder of the paper is organized as follows. In Section 2, we give precise definitions of the processes under study. In Section 3, we introduce the orthogonal polynomials that are associated with these processes, and note some relevant properties. The orthogonal-polynomial representation for the transition probabilities is described in Section 4. In Section 5, we discuss absorption probabilities and conditions for certain absorption, in preparation for the analysis in Section 6, where we study the quasi-stationary behaviour of the processes at hand. Our results comprise a characterization of quasistationary distributions for birth-death processes with killing, and some sufficient conditions for their existence. We conclude with some examples.

\section{Birth-death processes with killing}

We are concerned with a continuous-time Markov chain $\mathscr{X}:=\{X(t), t \geq 0\}$, taking values in the set $S:=\{-1\} \cup C$, where $C:=\{0,1,2, \ldots\}$ is an irreducible class and -1 is an absorbing state. Besides, $q_{-1, j}=0$ for all $j \in C$, the transition rates $q_{i j}$ of $\mathscr{X}$ satisfy

$$
q_{i j}=0, \quad i, j \in C,|i-j|>1,
$$

while

$$
\lambda_{i}:=q_{i, i+1}>0, \quad \mu_{i+1}:=q_{i+1, i}>0, \quad \gamma_{i}:=q_{i,-1} \geq 0, \quad i \in C .
$$

A process with these properties will be called a birth-death process with killing. The parameters $\lambda_{i}$ and $\mu_{i}$ are the birth rate and death rate, respectively, in state $i$, while $\gamma_{i}$ is the killing rate in state $i$, that is, the rate of absorption from state $i$ into state -1 . It will be convenient to define $\mu_{0}:=0$, indicating again that a transition from state 0 to state -1 is designated as "killing" rather than "death." The transition rates are conveniently assembled in the 
$q$-matrix $Q:=\left(q_{i j}, i, j \in S\right)$ of $\mathscr{X}$, where

$$
q_{-1,-1}=0, \quad q_{i i}=-\left(\lambda_{i}+\mu_{i}+\gamma_{i}\right), \quad i \in C .
$$

We write $\mathbb{P}_{i}(\cdot)$ for the probability measure of the process when $X(0)=i$, and let $\mathbb{P}_{m}(\cdot):=$ $\sum_{i} m_{i} \mathbb{P}_{i}(\cdot)$ for any initial distribution $m:=\left(m_{i}, i \in C\right)$.

Unless explicitly stated otherwise, we will assume that $\gamma_{i}>0$ for at least one state $i \in$ $C$, so that -1 is accessible from $C$. If the killing rates $\gamma_{i}$ are all zero except $\gamma_{0}>0$, then we are dealing with a pure birth-death process with an absorbing bottom state. We will sometimes refer to this case for comparison purposes.

Throughout the paper, we will assume that the process $\mathscr{X}$ is nonexplosive. A necessary and sufficient condition for nonexplosiveness of $\mathscr{X}$ in terms of the transition rates of $\mathscr{X}$ may be obtained from Chen et al. [2, Theorem 8], namely,

$$
\sum_{n=0}^{\infty} \frac{1}{\lambda_{n} \pi_{n}} \sum_{j=0}^{n}\left(1+\gamma_{j}\right) \pi_{j}=\infty
$$

where

$$
\pi_{0}:=1, \quad \pi_{n}:=\frac{\lambda_{0} \lambda_{1} \cdots \lambda_{n-1}}{\mu_{1} \mu_{2} \cdots \mu_{n}}, \quad n>0 .
$$

As is to be expected, this condition can be given the interpretation that either absorption at -1 is certain or the birth-death process obtained by setting $\gamma_{i}=0$ for all $i \in C$ is nonexplosive (see [12]). We will return to condition (2.4) later. At this point, it is important to note that, as a consequence of nonexplosiveness, the transition probability functions

$$
p_{i j}(t):=\mathbb{P}_{i}\{X(t)=j\}, \quad i, j \in S, t \geq 0,
$$

constitute the unique solution of the system of backward equations

$$
P^{\prime}(t)=Q P(t), \quad t \geq 0,
$$

and satisfy the forward equations

$$
P^{\prime}(t)=P(t) Q, \quad t \geq 0,
$$

with initial condition $P(0)=I$, where $P(t):=\left(p_{i j}(t), i, j \in S\right)$, and $I$ is the identity matrix.

\section{Orthogonal polynomials}

The transition rates of $\mathscr{X}$ determine a sequence of polynomials $\left\{R_{n}(x)\right\}$ through the recurrence relation

$$
\begin{gathered}
\lambda_{n} R_{n+1}(x)=\left(\lambda_{n}+\mu_{n}+\gamma_{n}-x\right) R_{n}(x)-\mu_{n} R_{n-1}(x), \quad n>0, \\
\lambda_{0} R_{1}(x)=\lambda_{0}+\gamma_{0}-x, \quad R_{0}(x)=1 .
\end{gathered}
$$


4 Quasi-stationary distributions for birth-death processes with killing

By letting

$$
P_{0}(x):=1, \quad P_{n}(x):=(-1)^{n} \lambda_{0} \lambda_{1} \cdots \lambda_{n-1} R_{n}(x), \quad n>0,
$$

we obtain the corresponding sequence of monic polynomials, which satisfy the recurrence relation

$$
\begin{gathered}
P_{n+1}(x)=\left(x-\lambda_{n}-\mu_{n}-\gamma_{n}\right) P_{n}(x)-\lambda_{n-1} \mu_{n} P_{n-1}(x), \quad n>0, \\
P_{1}(x)=x-\lambda_{0}-\gamma_{0}, \quad P_{0}(x)=1 .
\end{gathered}
$$

Since $\lambda_{n-1} \mu_{n}>0$ for $n>0$, it follows (see, e.g., Chihara [3, Theorems I.4.4 and II.3.1]) that $\left\{P_{n}(x)\right\}$, and hence $\left\{R_{n}(x)\right\}$, constitutes a sequence of orthogonal polynomials with respect to a bounded, positive Borel measure on $\mathbb{R}$.

If $\gamma_{n}=0$ for all $n$ except $\gamma_{0} \geq 0$, then $\mathscr{X}$ is a pure birth-death process and we know (from, e.g., [5], or the corollary to [3, Theorem I.9.1]) that the sequence $\left\{P_{n}(x)\right\}$ is orthogonal with respect to a measure on $[0, \infty)$. But it is not difficult to verify (see [11]) that there exist unique positive numbers $\tilde{\lambda}_{n}$ and $\tilde{\mu}_{n+1}, n \geq 0$, such that

$$
\lambda_{n}+\mu_{n}+\gamma_{n}=\tilde{\lambda}_{n}+\tilde{\mu}_{n}, \quad \lambda_{n} \mu_{n+1}=\tilde{\lambda}_{n} \tilde{\mu}_{n+1}, \quad n \geq 0,
$$

where $\tilde{\mu}_{0}:=0$. Substitution of (3.4) into (3.3) shows that also in the present, more general setting, the sequence $\left\{P_{n}(x)\right\}$, and hence the sequence $\left\{R_{n}(x)\right\}$, is orthogonal with respect to a bounded, positive Borel measure on $[0, \infty)$. It is of course no restriction of generality to assume that the measure has total mass 1 , so that it is a probability measure. Summarizing, there exists a probability measure $\psi$ on $[0, \infty)$, and positive constants $k_{j}$ such that

$$
k_{j} \int_{0}^{\infty} R_{i}(x) R_{j}(x) \psi(d x)=\delta_{i j}, \quad i, j \geq 0,
$$

where $\delta_{i j}$ is Kronecker's delta. Moreover, on applying [3, Theorem I.4.2(b)] to the sequence $\left\{P_{n}(x)\right\}$, it follows from (3.2) that $k_{j}=\pi_{j}$, the constants defined in (2.5).

It is well known that the polynomials $R_{n}(x)$ have real, positive zeros $x_{n 1}<x_{n 2}<\cdots<$ $x_{n n}, n \geq 1$, which are closely related to $\operatorname{supp}(\psi)$, the support of the measure $\psi$. In particular, we have

$$
\inf \operatorname{supp}(\psi)=\lim _{n \rightarrow \infty} x_{n 1}
$$

which exists, since the sequence $\left\{x_{n 1}\right\}$ is (strictly) decreasing (see, e.g., [3, Theorem II.4.5]). Considering that

$$
\lambda_{0} \lambda_{1} \cdots \lambda_{n-1} R_{n}(x)=\left(x_{n 1}-x\right)\left(x_{n 2}-x\right) \cdots\left(x_{n n}-x\right),
$$

it now follows that

$$
x \leq y \leq \inf \operatorname{supp}(\psi) \Longleftrightarrow R_{n}(x) \geq R_{n}(y)>0 \quad \forall n>0,
$$

a result that we will use in Section 6 . 
We conclude with some useful relations involving the polynomials $\left\{R_{n}(x)\right\}, n \geq 0$. Since $\lambda_{n-1} \pi_{n-1}=\mu_{n} \pi_{n}$, we can rewrite (3.1) as

$$
\begin{gathered}
\lambda_{n} \pi_{n}\left(R_{n+1}(x)-R_{n}(x)\right)=\lambda_{n-1} \pi_{n-1}\left(R_{n}(x)-R_{n-1}(x)\right)+\left(\gamma_{n}-x\right) \pi_{n} R_{n}(x), \quad n \geq 1, \\
\lambda_{0} \pi_{0}\left(R_{1}(x)-R_{0}(x)\right)=\left(\gamma_{0}-x\right) \pi_{0} R_{0}(x),
\end{gathered}
$$

so that

$$
\lambda_{n} \pi_{n}\left(R_{n+1}(x)-R_{n}(x)\right)=\sum_{j=0}^{n}\left(\gamma_{j}-x\right) \pi_{j} R_{j}(x), \quad n \geq 0 .
$$

Hence we can write

$$
R_{n}(x)=1+\sum_{k=0}^{n-1} \frac{1}{\lambda_{k} \pi_{k}} \sum_{j=0}^{k}\left(\gamma_{j}-x\right) \pi_{j} R_{j}(x), \quad n>0 .
$$

It follows in particular that

$$
R_{0}(0)=1, \quad R_{n}(0)=1+\sum_{k=0}^{n-1} \frac{1}{\lambda_{k} \pi_{k}} \sum_{j=0}^{k} \gamma_{j} \pi_{j} R_{j}(0), \quad n>0,
$$

so that $R_{n}(0)$ is increasing in $n$. From [12, Lemma 1], we know that

$$
\lim _{n \rightarrow \infty} R_{n}(0)=\infty \Longleftrightarrow \sum_{n=0}^{\infty} \frac{1}{\lambda_{n} \pi_{n}} \sum_{j=0}^{n} \gamma_{j} \pi_{j}=\infty,
$$

which will be used in Section 5 .

\section{Representation}

It has recently been shown in [11] that the transition probabilities of the process $\mathscr{X}$, insofar as they do not involve the absorbing state -1 , may be represented in the form

$$
p_{i j}(t)=\pi_{j} \int_{0}^{\infty} e^{-x t} R_{i}(x) R_{j}(x) \psi(d x), \quad i, j \in C, t \geq 0,
$$

where $\pi_{n}, n \geq 0$, are the constants defined in (2.5), $R_{n}(x), n \geq 0$, are the polynomials defined in (3.1), and $\psi$ is an orthogonalizing probability measure on $[0, \infty)$ for the polynomial sequence $\left\{R_{n}(x)\right\}$. This result generalizes Karlin's and McGregor's [5] classic representation theorem for the pure birth-death process. Note that by setting $t=0$, we regain (3.5), though it is not clear yet that the measure $\psi$ is unique. However, the transition probabilities $p_{i j}(t)$ constitute the unique solution to the backward equations (2.7) because of our nonexplosiveness assumption (2.4). Since the representation (4.1) reduces to

$$
p_{00}(t)=\int_{0}^{\infty} e^{-x t} \psi(d x), \quad t \geq 0
$$


if $i=j=0$, the uniqueness theorem for Laplace transforms therefore implies that the probability measure $\psi$ must be unique as well.

We note that our assumption $\gamma_{i}>0$ for at least one state $i \in C$ implies that the transition probabilities $p_{i j}(t), i, j \in C$, tend to zero as $t \rightarrow \infty$. Hence the representation (4.1) tells us that the measure $\psi$ cannot have a point mass at zero, so that $\psi$ is in fact a measure on $(0, \infty)$.

It is well known (see, e.g., [1]) that the transition probabilities $p_{i j}(t), i, j \in C$, have a common rate of convergence $\alpha$, satisfying

$$
\alpha=-\lim _{t \rightarrow \infty} \frac{1}{t} \log p_{i j}(t), \quad i, j \in C,
$$

and known as the decay parameter of $\mathscr{X}$ in $C$. It is obvious from (4.2) that

$$
\alpha=\inf \operatorname{supp}(\psi)
$$

so, in view of (3.8), $\alpha$ may also be characterised as

$$
\alpha=\max \left\{x \in \mathbb{R} \mid R_{n}(x)>0 \forall n \geq 0\right\},
$$

which will prove useful in what follows.

As an aside, we note that in the setting of the pure birth-death process, $\alpha$ equals $\alpha_{-1}$, the rate of convergence of the transition probabilities $p_{i,-1}(t), i \in C$, to their limits (see, e.g., [10]). In the present, more general setting, this is not necessarily true, but we do have $\alpha_{-1} \leq \alpha$. The latter result is implied by the inequality $p_{i j}(t) \leq 1-p_{i,-1}(t)$ if absorption is certain, and may be proven by considering a suitable transformation of the process if absorption is not certain (see, e.g., [12]).

\section{Absorption}

By $T$ we denote the absorption time, that is, the (possibly defective) random variable representing the time at which absorption in state -1 occurs. We let

$$
\tau_{i}:=\lim _{t \rightarrow \infty} \mathbb{P}_{i}\{T \leq t\}, \quad i \in C,
$$

and refer to $\tau_{i}$ as the (eventual) absorption probability when the initial state is $i$. It is shown in [12] that if

$$
R_{\infty}(0):=\lim _{n \rightarrow \infty} R_{n}(0)=\infty
$$

then $\tau_{i}=1$ for all $i \in C$ (so that absorption is certain for any initial distribution), whereas otherwise the eventual absorption probabilities satisfy

$$
\tau_{i}=1-\frac{R_{i}(0)}{R_{\infty}(0)}<1, \quad i \in C .
$$


In view of (3.13), a necessary and sufficient condition for certain absorption is therefore given by

$$
\sum_{n=0}^{\infty} \frac{1}{\lambda_{n} \pi_{n}} \sum_{j=0}^{n} \gamma_{j} \pi_{j}=\infty
$$

In the remainder of this paper, we will assume that (5.4) is satisfied so that absorption is certain. Note that this assumption is stronger than the nonexplosiveness assumption (2.4), maintained from the beginning.

\section{Quasi-stationarity}

6.1. Definitions and general results. A quasi-stationary distribution for $\mathscr{X}$ is a proper probability distribution $m:=\left(m_{j}, j \in C\right)$, such that for all $t \geq 0$,

$$
\mathbb{P}_{m}\{X(t)=j \mid T>t\}=m_{j}, \quad j \in C .
$$

That is, $m$ is a quasi-stationary distribution if the state probabilities of $\mathscr{X}$ at time $t$, conditional on the chain being in $C$ at time $t$, do not vary with $t$ when $m$ is chosen as initial distribution. We note that

$$
\mathbb{P}_{m}\{X(t)=j \mid T>t\}=\frac{\mathbb{P}_{m}\{X(t)=j\}}{\mathbb{P}_{m}\{T>t\}},
$$

while $\mathbb{P}_{m}\{X(t)=j\} \rightarrow 0$ as $t \rightarrow \infty$ for all $j \in C$ and any initial distribution $m$. So, $m$ can be a quasi-stationary distribution only if $\mathbb{P}_{m}\{T>t\} \rightarrow 0$ as $t \rightarrow \infty$, that is, if absorption is certain, our assumption throughout this section.

It will be convenient to introduce another concept. Namely, a proper probability distribution $\left(m_{j}, j \in C\right)$ over the nonabsorbing states is called $x$-invariant for $Q$ (the $q$-matrix of $\mathscr{L}$ ) for some real $x$ if

$$
\sum_{i \in C} m_{i} q_{i j}=-x m_{j}, \quad j \in C
$$

The notions of $x$-invariant distribution and quasi-stationary distribution are intimately related. Indeed, combining Proposition 3.1 of Nair and Pollett [6] and Theorem 1 and Corollary 1 of Pollett and Vere-Jones [7], and recalling that, in our setting, the transition probabilities satisfy the forward equations (2.8), we can state the following.

Theorem 6.1 (see $[6,7]$ ). Let $\mathscr{X}$ be a birth-death process with killing such that absorption at -1 is certain. If $m:=\left(m_{j}, j \in C\right)$ is a quasi-stationary distribution, then $m$ is $x$-invariant for $Q$ for some $x>0$. Conversely, if $m$ is $x$-invariant for $Q$, then $m$ is a quasi-stationary distribution if and only if

$$
x=\sum_{j \in C} m_{j} \gamma_{j}
$$

We note that summing (6.3) over all $j \in C$ results in (6.4) if the interchange of summation would be justified, which, however, is not the case in general. 
8 Quasi-stationary distributions for birth-death processes with killing

Vere-Jones [13] showed that if $\left(m_{j}, j \in C\right)$ is a quasi-stationary distribution, and hence $x$-invariant for $\mathrm{Q}$ for some $x$, then $x$ must be in the interval $0<x \leq \alpha$, where $\alpha$ is the decay parameter of $\mathscr{X}$ in $C$ defined in (4.3). It follows that, besides certain absorption, $\alpha>0$ is necessary for the existence of a quasi-stationary distribution.

In summary, if $\alpha>0$ and absorption is certain, then, in order to find all quasi-stationary distributions for $\mathscr{L}$, we have to find all proper distributions $\left(m_{j}, j \in C\right)$ which constitute a solution of (6.3) for some $x, 0<x \leq \alpha$, and satisfy (6.4).

6.2. Quasi-stationary distributions. Considering the recurrence relation (3.1) for the polynomial sequence $\left\{R_{n}(x)\right\}$, the solution of the system of $(6.3)$ is readily seen to be given by

$$
m_{j}=m_{0} \pi_{j} R_{j}(x), \quad j \in C,
$$

where $m_{0}$ is some constant. To obtain all quasi-stationary distributions, we thus have to find out for which values of $x, 0<x \leq \alpha$, the quantities $m_{j}$ of (6.5) constitute a proper distribution with an appropriate choice of $m_{0}$, and satisfy (6.4). So the following three conditions have to be satisfied.

(i) We must have $m_{j} \geq 0$ for all $j$, and hence $R_{j}(x) \geq 0$ for all $j$. But this is a consequence of our assumption $x \leq \alpha$, which, by (4.5), implies that $R_{j}(x)>0$ for all $j$.

(ii) The sum $\sum_{j \in C} \pi_{j} R_{j}(x)$ must be finite, so that $\left(m_{j}, j \in C\right)$ becomes a proper distribution by choosing $m_{0}^{-1}=\sum_{j \in C} \pi_{j} R_{j}(x)$.

(iii) Condition (6.4) must be satisfied, that is, if the previous requirements are met, we must have

$$
x \sum_{j \in C} \pi_{j} R_{j}(x)=\sum_{j \in C} \gamma_{j} \pi_{j} R_{j}(x)
$$

Summarizing the preceding, we can state the following theorem.

Theorem 6.2. Let $\mathscr{X}$ be a birth-death process with killing such that absorption at -1 is certain. If $\alpha=0$, there is no quasi-stationary distribution for $\mathscr{X}$. If $\alpha>0$, then $\left(m_{j}, j \in C\right)$ is a quasi-stationary distribution for $\mathscr{X}$ if and only if there is a real number $x, 0<x \leq \alpha$, such that

$$
x \sum_{j \in C} \pi_{j} R_{j}(x)=\sum_{j \in C} \gamma_{j} \pi_{j} R_{j}(x)<\infty
$$

and $m_{j}=m_{j}(x), j \in C$, where

$$
m_{j}(x):=m_{0}(x) \pi_{j} R_{j}(x), \quad j \in C, \quad m_{0}(x)^{-1}:=\sum_{j \in C} \pi_{j} R_{j}(x)
$$

To verify whether (6.7) holds, the next lemma, which follows immediately from (3.10) and the fact that $R_{j}(x)>0$ for $x \leq \alpha$, is helpful. 
Lemma 6.3. Let $0<x \leq \alpha$. Then (6.7) is satisfied if and only if both

$$
\sum_{j \in C} \pi_{j} R_{j}(x)<\infty \quad \text { or } \quad \sum_{j \in C} \gamma_{j} \pi_{j} R_{j}(x)<\infty
$$

and

$$
\lim _{j \rightarrow \infty} \lambda_{j} \pi_{j}\left(R_{j+1}(x)-R_{j}(x)\right)=0 .
$$

Unfortunately, it does not seem possible to give a general condition in terms of the rates of the process for (6.9) and (6.10) to be valid. However, more can be said by imposing some additional restrictions on the rates. In the following subsections, some special cases will be therefore discussed.

6.3. Special case: finitely many positive killing rates. Let us first consider the situation in which $\gamma_{i}>0$ for only finitely many states $i \in C$. In this case, (6.9) is trivially satisfied. Actually, both sums in (6.9) converge, as appears from the next lemma.

Lemma 6.4. Let $\mathscr{X}$ be a birth-death process with killing for which absorption at -1 is certain and $\gamma_{i}>0$ for only finitely many states $i \in C$. Then $\sum_{j \in C} \pi_{j} R_{j}(x)<\infty$ for all $x$ in the interval $0<x \leq \alpha$.

Proof. When $\gamma_{i}>0$ for only finitely many states $i \in C$, our assumption (5.4) reduces to

$$
\sum_{k=0}^{\infty} \frac{1}{\lambda_{k} \pi_{k}}=\infty
$$

Now let $0<x \leq \alpha$ and suppose $\sum_{j \in C} \pi_{j} R_{j}(x)$ diverges. Since $\sum_{j \in C} \gamma_{j} \pi_{j} R_{j}(x)$ converges, we then have

$$
\sum_{j=0}^{k}\left(\gamma_{j}-x\right) \pi_{j} R_{j}(x) \longrightarrow-\infty \quad \text { as } k \longrightarrow \infty
$$

so that, by (3.11) and (6.11), $R_{j}(x)$ must be negative for $j$ sufficiently large. But this is a contradiction, since, by (4.5), $R_{j}(x)>0$ for all $j$ if $x \leq \alpha$. So the sum $\sum_{j \in C} \pi_{j} R_{j}(x)$ must be finite.

We conclude that $\left(m_{j}(x), j \in C\right)$, where $m_{j}(x)$ denotes the quantity defined in (6.8), constitutes a proper distribution for all $x$ in the interval $0<x \leq \alpha$. However, it is not necessarily true that (6.10), and hence (6.7), is satisfied. In the special case of a pure birth-death process $\left(\gamma_{i}=0\right.$ for all $i>0$ and $\left.\gamma_{0}>0\right)$, a necessary and sufficient condition for (6.7) to be valid for all $x$ in the interval $0<x \leq \alpha$ is that the sum

$$
\sum_{n=0}^{\infty} \frac{1}{\lambda_{n} \pi_{n}} \sum_{j=n+1}^{\infty} \pi_{j}
$$


should be divergent (see [9, Theorem 3.2]). The proof of this result relies on the fact that the polynomials

$$
\lambda_{n} \pi_{n}\left(R_{n+1}(x)-R_{n}(x)\right), \quad n \geq 0,
$$

are themselves orthogonal with respect to a probability measure on $[0, \infty)$. This property is lost as soon as one leaves the setting of the pure birth-death process, but if $\gamma_{i}>0$ for only finitely many states $i \in C$, we can get around this problem.

Theorem 6.5. Let $\mathscr{X}$ be a birth-death process with killing for which absorption at -1 is certain and $\gamma_{i}>0$ for only finitely many states $i \in C$. If $\alpha>0$ and the sum (6.13) diverges, then $\left(m_{j}(x), j \in C\right)$, with $m_{j}(x)$ given by (6.8), constitutes a quasi-stationary distribution for all $x$ in the interval $0<x \leq \alpha$.

We have relegated the proof of this theorem to the appendix, since it requires techniques which are not related to the central issues of this section.

Let us now assume that the sum (6.13) is convergent. In a pure birth-death process, we must then have $\alpha>0$, and there is precisely one quasi-stationary distribution, namely, $\left(m_{j}(\alpha), j \in C\right)$ (see [9, Theorem 3.2] again). In the present, more general setting, we cannot exclude the possibilities that $\alpha=0$ and, if $\alpha>0$, that there are several values of $x$ in the interval $0<x \leq \alpha$ such that $\left(m_{j}(x), j \in C\right)$ constitutes a quasi-stationary distribution (these values of $x$ would correspond to zeros of an entire function), but in any case, we can show the following.

Theorem 6.6. Let $\mathscr{X}$ be a birth-death process with killing for which absorption at -1 is certain and $\gamma_{i}>0$ for only finitely many states $i \in C$. If $\alpha>0$ and the sum (6.13) converges, then $\left(m_{j}(\alpha), j \in C\right)$, with $m_{j}(\alpha)$ given by (6.8), constitutes a quasi-stationary distribution.

Proof. From [12, Theorem 2], we know that (6.6) is satisfied for $x=\alpha$, although both sums may be infinite. However, Lemma 6.4 tells us that under the prevailing conditions, the sums must be finite. The result follows by Theorem 6.2.

6.4. Special case: bounded birth and death rates. We will next consider the setting in which

$$
\lambda_{i}+\mu_{i} \leq M<\infty, \quad i \in C,
$$

for some $M \in \mathbb{R}^{+}$. As usual, $m_{j}(x)$ denotes the quantity defined in (6.8) and we will tacitly assume that $0<x \leq \alpha$.

Since $\lambda_{j} \pi_{j}=\mu_{j+1} \pi_{j+1}$, we have

$$
\lambda_{j} \pi_{j}\left(R_{j+1}(x)-R_{j}(x)\right)=\mu_{j+1} \pi_{j+1} R_{j+1}(x)-\lambda_{j} \pi_{j} R_{j}(x)
$$

which tends to zero if $\lambda_{j}$ and $\mu_{j}$ are bounded and $\sum_{j \in C} \pi_{j} R_{j}(x)$ converges. So, by Lemma 6.3, the condition (6.7) for $\left(m_{j}(x), j \in C\right)$ to be a quasi-stationary distribution is fulfilled if $\sum_{j \in C} \pi_{j} R_{j}(x)<\infty$. But we can do somewhat better as follows. 
Theorem 6.7. Let $\mathscr{X}$ be a birth-death process with killing satisfying (6.15), for which absorption at -1 is certain. If $0<x \leq \alpha$ and $\sum_{j \in C} \pi_{j} R_{j}(x)<\infty$, then $\left(m_{j}(y), j \in C\right)$ is a quasi-stationary distribution for all $y$ in the interval $x \leq y \leq \alpha$.

Proof. We observe from (3.8) that $0<\sum_{j \in C} \pi_{j} R_{j}(y) \leq \sum_{j \in C} \pi_{j} R_{j}(x)$ if $x \leq y \leq \alpha$, so $\left(m_{j}(y), j \in C\right)$ is a quasi-stationary distribution for all $y$ in the interval $x \leq y \leq \alpha$ if $\sum_{j \in C} \pi_{j} R_{j}(x)<\infty$.

We conclude that if absorption is certain, $\alpha>0$, and the birth and death rates are bounded, then either there is no quasi-stationary distribution (if $\sum_{j \in C} \pi_{j} R_{j}(x)$ diverges) or $\left(m_{j}(x), j \in C\right)$ constitutes a quasi-stationary distribution for all $x$ in an interval of the type $0<a \leq x \leq \alpha$ (allowing for $a=\alpha$ ), or of the type $0 \leq a<x \leq \alpha$. If there are infinitely many quasi-stationary distributions, that is, $a<\alpha$, then $\left(m_{j}(x), j \in C\right)$ need not be a quasi-stationary distribution for all $x$ in the interval $0<x \leq \alpha$, so $a$ can be strictly positive. An example of the latter type of behaviour is given in the next subsection. Specific settings in which there is precisely one quasi-stationary distribution, or no quasi-stationary distribution at all, occur in the second example of the next subsection.

6.5. Examples. We will first construct a process such that a quasi-stationary distribution which is $x$-invariant exists if and only if $a<x \leq \alpha$ for some $a>0$. Indeed, let $\mathscr{X}$ be a birth-death process with killing with birth, death, and killing rates $\lambda_{i}, \mu_{i+1}$, and $\gamma_{i}, i \in C$, respectively, $q$-matrix $Q$ and decay parameter $\alpha$. Next, choose $\gamma>0$ and let $\tilde{\mathscr{X}}$ be the birthdeath process with killing with transition rates $\tilde{\lambda}_{i}:=\lambda_{i}, \tilde{\mu}_{i+1}:=\mu_{i+1}, i \in C$, and

$$
\tilde{\gamma}_{i}:=\gamma+\gamma_{i}, \quad i \in C
$$

and $q$-matrix $\widetilde{Q}$. One might interpret $\tilde{\mathscr{L}}$ as the superposition of $\mathscr{L}$ and an independent Poisson killing process of rate $\gamma$. Obviously, the transition probabilities of $\tilde{\mathscr{X}}$ and $\mathscr{X}$ are related as

$$
\widetilde{P}_{i j}(t)=e^{-\gamma t} P_{i j}(t), \quad i, j \in C, t \geq 0
$$

whence the decay parameter $\tilde{\alpha}$ of $\tilde{\mathscr{X}}$ satisfies $\tilde{\alpha}=\gamma+\alpha$. It is evident from (6.3) and Theorem 6.1 that an $x$-invariant quasi-stationary distribution for $\mathscr{X}$ is a $(\gamma+x)$-invariant quasistationary distribution for $\tilde{\mathscr{X}}$, and vice versa. Now, if we choose $\mathscr{X}$ such that for each $x$ in the interval $0<x \leq \alpha$ there exists a quasi-stationary distribution (e.g., by letting $\mathscr{X}$ be a suitable pure birth-death process, cf. [9]), then for each $\tilde{x}$ in the interval $\gamma<\tilde{x} \leq \tilde{\alpha}$ there exists an $\tilde{x}$-invariant quasi-stationary distribution for $\tilde{\mathscr{X}}$, but there are no $\tilde{x}$-invariant quasi-stationary distributions for $\tilde{\mathscr{X}}$ with $\tilde{x} \leq \gamma$, since an $x$-invariant quasi-stationary distribution for $\mathscr{X}$ must have $x>0$. Thus $\tilde{\mathscr{X}}$ has the required property, with $a=\gamma$.

Our final example is the process $\mathscr{X}$ with birth, death, and killing rates

$$
\lambda_{i}=\lambda, \quad \mu_{i}=\mu \rrbracket_{\{i>0\}}, \quad \gamma_{i}=\gamma \rrbracket_{\{i>0\}}, \quad i \in C,
$$

for some constants $\lambda>0, \mu>0$, and $\gamma>0$, where $\rrbracket_{E}$ denotes the indicator function of an event $E$. So killing may occur from any state except state 0 . From [12, Section 6], we find 
12 Quasi-stationary distributions for birth-death processes with killing

that the decay parameter for this process is given by

$$
\alpha= \begin{cases}\frac{\lambda \gamma}{\mu+\gamma} & \text { if } \mu+\gamma \geq \sqrt{\lambda \mu}, \\ \gamma+(\sqrt{\lambda}-\sqrt{\mu})^{2} & \text { if } \mu+\gamma<\sqrt{\lambda \mu}\end{cases}
$$

while

$$
\pi_{j} R_{j}(x)=(-1)^{j}\left(\frac{\lambda}{\mu}\right)^{j / 2}\left(U_{j}(y)+\eta U_{j-1}(y)\right), \quad j \geq 0,
$$

where

$$
y:=\frac{x-\lambda-\mu-\gamma}{2 \sqrt{\lambda \mu}}, \quad \eta:=\frac{\mu+\gamma}{\sqrt{\lambda \mu}},
$$

and $U_{j}(\cdot)$ denotes the $j$ th Chebyshev polynomial of the second kind, that is,

$$
U_{j}(y)=\frac{z^{j+1}-z^{-(j+1)}}{z-z^{-1}}, \quad j \geq 0,
$$

with $z$ such that $y=(1 / 2)\left(z+z^{-1}\right)$. Evidently, absorption is certain. Moreover, since $\lambda_{i}$ and $\mu_{i}$ are bounded, we can employ Theorem 6.7 and conclude that we must determine all $x$ such that $0<x \leq \alpha$ and $\sum_{j \in C} \pi_{j} R_{j}(x)<\infty$, in order to find all quasi-stationary distributions.

So let $0<x \leq \alpha$. Considering that

$$
\frac{\lambda \gamma}{\mu+\gamma}=\lambda+\mu+\gamma-\sqrt{\lambda \mu}\left(\eta+\eta^{-1}\right) \leq \gamma+(\sqrt{\lambda}-\sqrt{\mu})^{2}
$$

we have $0<x \leq \lambda+\mu+\gamma-2 \sqrt{\lambda \mu}$, and hence $y=(1 / 2)\left(z+z^{-1}\right) \leq-1$. It is therefore no restriction of generality to assume $z \leq-1$ (and hence $-1 \leq z^{-1}<0$ ). Moreover, we can write

$$
\pi_{j} R_{j}(x)= \begin{cases}\left(\frac{\lambda}{\mu}\right)^{j / 2}\left\{\frac{(-z)^{j}(z+\eta)-(-z)^{-j}\left(\eta+z^{-1}\right)}{z-z^{-1}}\right\} & \text { if } z<-1, \\ \left(\frac{\lambda}{\mu}\right)^{j / 2}\{1+(1-\eta) j\} & \text { if } z=-1,\end{cases}
$$

so that $\sum_{j \in C} \pi_{j} R_{j}(x)$ diverges unless either $z \neq-\eta$ and $-z \sqrt{\lambda / \mu}<1$, or $z=-\eta$ and $\eta^{-1} \sqrt{\lambda / \mu}=\lambda /(\mu+\gamma)<1$. We now discern the following three cases.

(i) If $\lambda \geq \mu+\gamma$, then $-z \sqrt{\lambda / \mu}>1$ and $\eta^{-1} \sqrt{\lambda / \mu} \geq 1$. Hence $\sum_{j \in C} \pi_{j} R_{j}(x)$ diverges.

(ii) If $\lambda<\mu+\gamma$ (and hence $\eta>1$ ) and $x<\alpha$, then $y<-(1 / 2)\left(\eta+\eta^{-1}\right)$ and hence $z<$ $-\eta$, so that $z \neq-\eta$ and $-z \sqrt{\lambda / \mu}>1$. Again it follows that $\sum_{j \in C} \pi_{j} R_{j}(x)$ diverges.

(iii) If $\lambda<\mu+\gamma, x=\alpha$, then $z=-\eta$ and $\eta^{-1} \sqrt{\lambda / \mu}<1$. So now we have $\sum_{j \in C} \pi_{j} R_{j}(x)<\infty$. 
Concluding, there is no quasi-stationary distribution if $\lambda \geq \mu+\gamma$, and there is precisely one quasi-stationary distribution $\left(m_{j}, j \in C\right)$, where

$$
m_{j}=m_{j}(\alpha)=\left(1-\frac{\lambda}{\mu+\gamma}\right)\left(\frac{\lambda}{\mu+\gamma}\right)^{j}, \quad j \geq 0,
$$

if $\lambda<\mu+\gamma$, that is, $\alpha<\gamma$. The existence of a quasi-stationary distribution under these circumstances is predicted by the discrete-state-space analogue of [8, Theorem 3.4].

\section{Appendix}

\section{A. Proof of Theorem 6.5}

We start off with collecting some preliminary results. Given the polynomials $R_{n}(x), n \geq 0$, of (3.1), we define the associated polynomials of order $m, m>0$, by the recurrence relation

$$
\begin{gathered}
\lambda_{n}^{(m)} R_{n+1}^{(m)}(x)=\left(\lambda_{n}^{(m)}+\mu_{n}^{(m)}+\gamma_{n}^{(m)}-x\right) R_{n}^{(m)}(x)-\mu_{n}^{(m)} R_{n-1}^{(m)}(x), \quad n>0, \\
\lambda_{0}^{(m)} R_{1}^{(m)}(x)=\lambda_{0}^{(m)}+\mu_{0}^{(m)}+\gamma_{0}^{(m)}-x, \quad R_{0}^{(m)}(x)=1,
\end{gathered}
$$

where

$$
\lambda_{n}^{(m)}:=\lambda_{m+n}, \quad \mu_{n}^{(m)}:=\mu_{m+n}, \quad \gamma_{n}^{(m)}:=\gamma_{m+n}, \quad n \geq 0
$$

Evidently, the polynomials $R_{n}^{(m)}(x), n \geq 0$, correspond to a birth-death process with killing $\mathscr{L}^{(m)}$ (with killing rate $\mu_{0}^{(m)}+\gamma_{0}^{(m)}$ in state 0 ), and are therefore orthogonal with respect to a positive measure $\psi^{(m)}$ on $[0, \infty)$. We define $\xi^{(m)}:=\inf \operatorname{supp}\left(\psi^{(m)}\right)$, and note from (e.g., [3, Theorem III.4.2]) that

$$
\xi^{(m)} \leq \xi^{(m+1)}, \quad m \geq 0
$$

where $\xi^{(0)}:=\inf \operatorname{supp}(\psi)$, and so, by $(4.4), \xi^{(0)}=\alpha$.

Next, it follows readily by induction on $n$ that for all $m>0$, we have

$$
R_{m+n}(x)=R_{m}(x) R_{n}^{(m)}(x)-\frac{\mu_{m}}{\lambda_{m}} R_{m-1}(x) R_{n-1}^{(m+1)}(x), \quad n>0 .
$$

Defining $\pi_{n}^{(m)}$ by analogy with (2.5), we have $\pi_{m+n}=\pi_{m} \pi_{n}^{(m)}$, and hence the previous equation implies that for all $m>0$ and $n>0$,

$$
\begin{aligned}
& \lambda_{m+n} \pi_{m+n}\left(R_{m+n+1}(x)-R_{m+n}(x)\right) \\
& \quad=a(x)\left[\lambda_{n}^{(m)} \pi_{n}^{(m)}\left(R_{n+1}^{(m)}(x)-R_{n}^{(m)}(x)\right)\right]-b(x)\left[\lambda_{n-1}^{(m+1)} \pi_{n-1}^{(m+1)}\left(R_{n}^{(m+1)}(x)-R_{n-1}^{(m+1)}(x)\right)\right],
\end{aligned}
$$

where

$$
a(x):=\pi_{m} R_{m}(x), \quad b(x):=\frac{\lambda_{m-1}}{\mu_{m+1}} \pi_{m-1} R_{m-1}(x) .
$$


14 Quasi-stationary distributions for birth-death processes with killing

Finally, considering that

$$
\sum_{n=m}^{\infty} \frac{1}{\lambda_{n} \pi_{n}} \sum_{j=n+1}^{\infty} \pi_{j}=\sum_{n=0}^{\infty} \frac{1}{\lambda_{n}^{(m)} \pi_{n}^{(m)}} \sum_{j=n+1}^{\infty} \pi_{j}^{(m)},
$$

we see that divergence of (6.13) is equivalent to

$$
\sum_{n=0}^{\infty} \frac{1}{\lambda_{n}^{(m)} \pi_{n}^{(m)}} \sum_{j=n+1}^{\infty} \pi_{j}^{(m)}=\infty
$$

for any $m>0$.

Now suppose $\gamma_{i}=0$ for $i \geq N$. Then, for $m \geq N$, the orthogonal-polynomial sequence $\left\{R_{n}^{(m)}(x)\right\}$ corresponds to a pure birth-death process. Therefore, if the sum (6.13) diverges, and hence (A.8) is satisfied, then, by [9, Theorem 3.2], we have, for all $m \geq N$ and $0<x \leq \xi^{(m)}$,

$$
x \sum_{j=0}^{\infty} \pi_{j}^{(m)} R_{j}^{(m)}(x)=\mu_{0}^{(m)},
$$

which is $(6.7)$ in terms of $\mathscr{X}^{(m)}$. (Recall that the killing rate of $\mathscr{X}^{(m)}$ in state 0 is $\mu_{0}^{(m)}+\gamma_{0}^{(m)}$.) Lemma 6.3 subsequently implies that, for all $m \geq N$ and $0<x \leq \xi^{(m)}$,

$$
\lambda_{n}^{(m)} \pi_{n}^{(m)}\left(R_{n+1}^{(m)}(x)-R_{n}^{(m)}(x)\right) \longrightarrow 0 \quad \text { as } n \longrightarrow \infty .
$$

Choosing $m=N$ in (A.5), and applying (A.10) and (A.3), we see that (6.10), and hence by Lemma 6.3, (6.7), is satisfied for all $x$ in the interval $0<x \leq \xi^{(0)}=\alpha$. The statement of Theorem 6.5 now follows from Theorem 6.2.

\section{Acknowledgments}

This paper was written while the second author visited Durham University holding a Grey College Fellowship. He thanks Grey College and the Department of Mathematical Sciences of Durham University for the hospitality extended during this visit.

\section{References}

[1] W. J. Anderson, Continuous-Time Markov Chains, Springer Series in Statistics, Springer, New York, 1991.

[2] A. Chen, P. Pollett, H. Zhang, and B. Cairns, Uniqueness criteria for continuous-time Markov chains with general transition structures, Advances in Applied Probability 37 (2005), no. 4, 10561074.

[3] T. S. Chihara, An Introduction to Orthogonal Polynomials, Gordon and Breach, New York, 1978.

[4] P. Coolen-Schrijner and E. A. van Doorn, Quasi-stationary distributions for a class of discrete-time Markov chains, to appear in Methodology and Computing in Applied Probability.

[5] S. Karlin and J. L. McGregor, The differential equations of birth-and-death processes, and the Stieltjes moment problem, Transactions of the American Mathematical Society 85 (1957), no. 2, 489546. 
[6] M. G. Nair and P. K. Pollett, On the relationship between $\mu$-invariant measures and quasistationary distributions for continuous-time Markov chains, Advances in Applied Probability 25 (1993), no. 1, 82-102.

[7] P. K. Pollett and D. Vere-Jones, A note on evanescent processes, The Australian Journal of Statistics 34 (1992), no. 3, 531-536.

[8] D. Steinsaltz and S. N. Evans, Quasi-stationary distributions for one-dimensional diffusions with killing, to appear in Transactions of the American Mathematical Society.

[9] E. A. van Doorn, Quasi-stationary distributions and convergence to quasi-stationarity of birthdeath processes, Advances in Applied Probability 23 (1991), no. 4, 683-700.

[10] - On associated polynomials and decay rates for birth-death processes, Journal of Mathematical Analysis and Applications 278 (2003), no. 2, 500-511.

[11] E. A. van Doorn and A. I. Zeifman, Birth-death processes with killing, Statistics \& Probability Letters 72 (2005), no. 1, 33-42.

[12] Extinction probability in a birth-death process with killing, Journal of Applied Probability 42 (2005), no. 1, 185-198.

[13] D. Vere-Jones, Some limit theorems for evanescent processes, The Australian Journal of Statistics 11 (1969), 67-78.

Pauline Coolen-Schrijner: Department of Mathematical Sciences, Durham University,

DH1 3LE Durham, UK

E-mail address: pauline.schrijner@durham.ac.uk

Erik A. van Doorn: Department of Applied Mathematics, University of Twente, P.O. Box 217, 7500 AE Enschede, The Netherlands

E-mail address: e.a.vandoorn@utwente.nl 


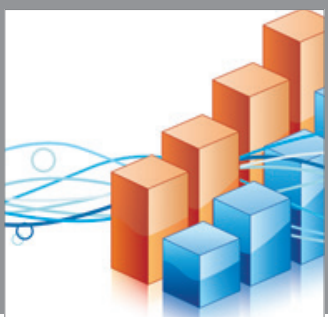

Advances in

Operations Research

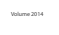

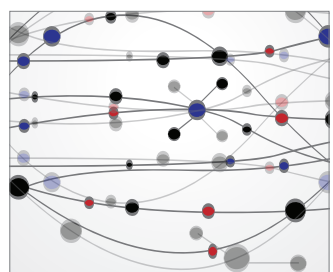

\section{The Scientific} World Journal
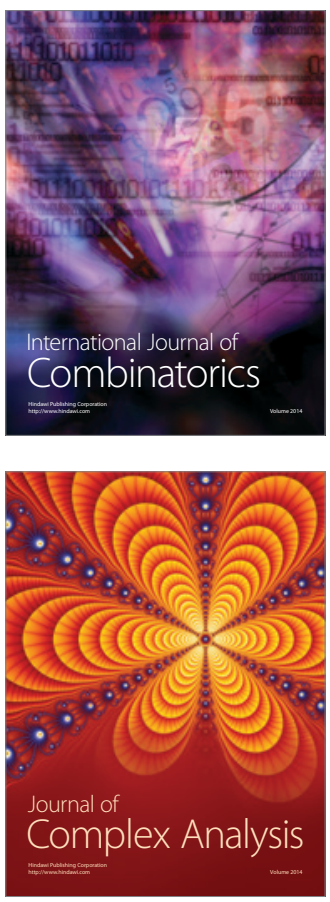

International Journal of

Mathematics and

Mathematical

Sciences
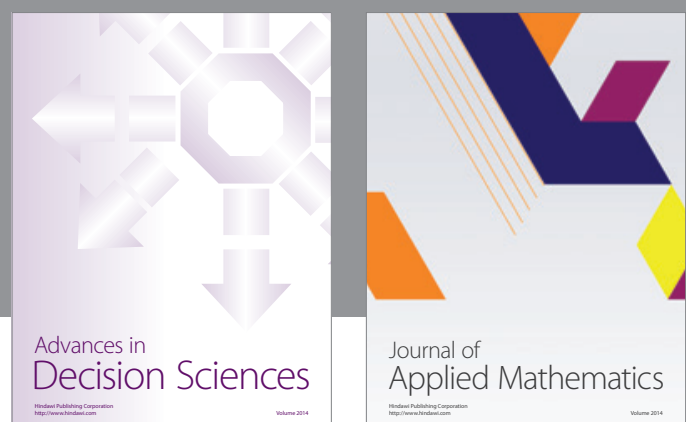

Journal of

Applied Mathematics
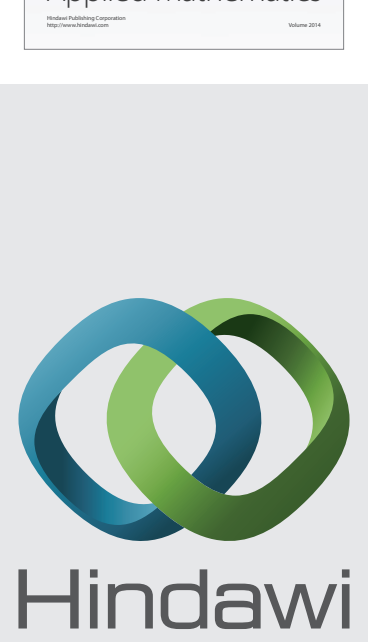

Submit your manuscripts at http://www.hindawi.com
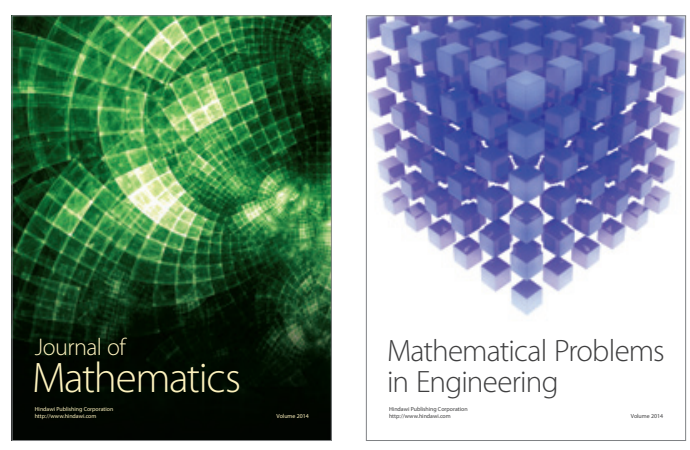

Mathematical Problems in Engineering
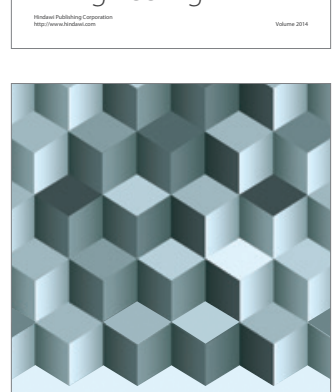

Journal of

Function Spaces
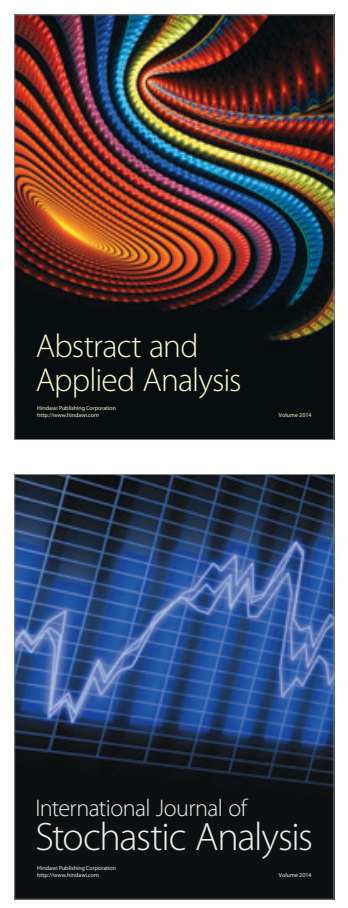

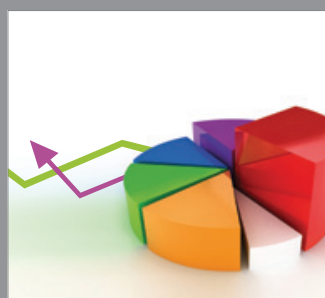

ournal of

Probability and Statistics

Promensencen
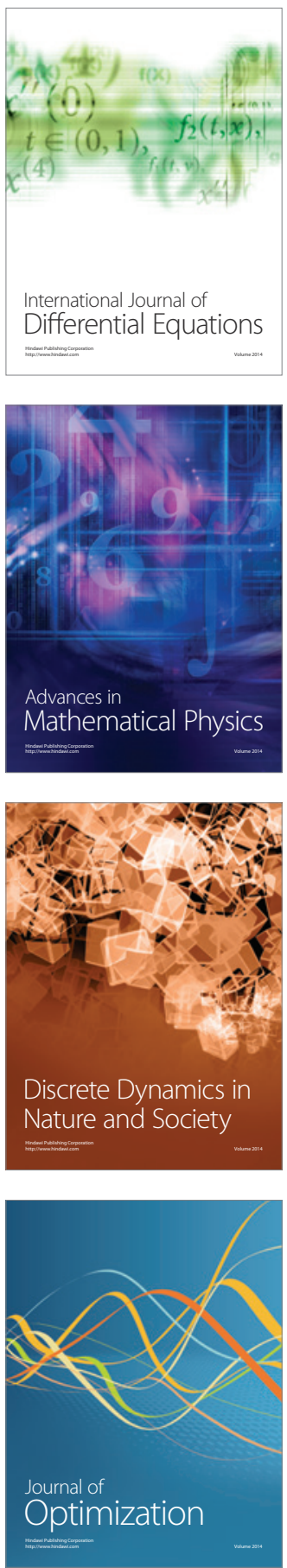